\title{
Correction to: Involvement of isoorientin in the antidepressant bioactivity of a flavonoid-rich extract from Passiflora edulis $\mathbf{f}$. flavicarpa leaves
}

Jovelina S. F. Alves ${ }^{1}$ • José I. Marques ${ }^{1} \cdot$ Daniel P. Demarque $^{2} \cdot$ Layse R. F. Costa $^{3}$ • Juliano G. Amaral ${ }^{2}$. Norberto P. Lopes ${ }^{2}$ - Arnóbio A. da Silva-Júnior ${ }^{1}$ - Luiz A. L. Soares ${ }^{4}$ - Elaine C. Gavioli ${ }^{3}$. Leandro De S. Ferreira ${ }^{1} \cdot$ Silvana M. Zucolotto ${ }^{1}$

Published online: 27 March 2020

(C) Sociedade Brasileira de Farmacognosia 2020

Correction to: Revista Brasileira de Farmacognosia https://doi.org/10.1007/s43450-020-00003-x

This article was updated to include the chemical structures.

The online version of the original article can be found at https://doi.org/ $10.1007 / \mathrm{s} 43450-020-00003-\mathrm{x}$

Silvana M. Zucolotto

silvanazucolotto@ufrnet.br

1 Centro de Ciências da Saúde, Programa de Pós-graduação em Ciências da Saúde, Universidade Federal do Rio Grande do Norte, Natal, RN, Brazil

2 Departamento de Física e Química, Faculdade de Ciências Farmacêuticas de Ribeirão Preto, Universidade de São Paulo, Ribeirão Preto, SP, Brazil

3 Centro de Ciências da Saúde, Departamento de Biofísica e Farmacologia, Universidade Federal do Rio Grande do Norte, Natal, RN, Brazil

4 Departamento de Ciências Farmacêuticas, Universidade Federal de Pernambuco, Recife, PE, Brazil 